\title{
Energy relaxation versus spectral diffusion of the OH-stretching vibration of HOD in liquid-to-supercritical deuterated water
}

\author{
Dirk Schwarzer \\ Max-Planck-Institut für biophysikalische Chemie, Abteilung Spektroskopie und Photochemische Kinetik, \\ Am Fassberg 11, 37077 Göttingen, Germany \\ Jörg Lindner and Peter Vöhringer ${ }^{a)}$ \\ Rheinische Friedrich-Wilhelms-Universität, Institut für Physikalische und Theoretische Chemie, \\ Wegelerstraße 12, 53115 Bonn, Germany
}

(Received 29 June 2005; accepted 13 September 2005; published online 25 October 2005)

\begin{abstract}
The dynamics of vibrational energy relaxation (VER) of the OH-stretching vibration of HOD in liquid-to-supercritical heavy water is studied as a function of temperature and solvent density by femtosecond mid-infrared spectroscopy. Using the dielectric constant of the fluid both, the $\mathrm{OH}$-stretching absorption frequency and the VER rate, can be correlated phenomenologically with the average hydrogen-bond connectivity within the random $\mathrm{D}_{2} \mathrm{O}$ network. This correlation enables the identification of thermodynamic conditions under which spectral diffusion due to hydrogen-bond breakage/formation is much faster than VER. (C) 2005 American Institute of Physics.
\end{abstract}

[DOI: $10.1063 / 1.2110087]$

The famous anomalies found in the thermodynamic quantities of liquid water ${ }^{1}$ can be connected to the formation of an extended network of hydrogen bonds ( $\mathrm{H}$ bonds), which is structurally and dynamically highly random in nature. Exploring the geometric distribution of $\mathrm{H}$ bonds and determining their structural relaxations are therefore the keys to a comprehensive understanding of this highly peculiar "solvent" of life.

Recently, femtosecond vibrational spectroscopy has proven to be highly instrumental in revealing molecular dynamical processes in liquid water. ${ }^{2-11}$ In particular, the dynamics of vibrational energy relaxation (VER) following an initial $\mathrm{OH}$-stretching ${ }^{5,12-15}$ or $\mathrm{HOH}$-bending ${ }^{16,17}$ excitation have been recorded directly in the time domain. For the neat liquid, VER at early times is dictated by intra- and intermolecular resonance phenomena resulting eventually in a complete delocalization of an initial vibrational excitation over a large number of neighboring particles accompanied by an ultrarapid loss of memory of the transition dipole orientation. ${ }^{10}$

Contrary to the neat liquid, vibrational delocalization is suppressed in the VER of HOD impurities in liquid water. The isotope substitution lifts inter- and intramolecular resonances thereby facilitating a spectroscopic separation of vibrational modes of the solute from those of the solvent. Particularly intriguing were experiments $\mathrm{s}^{3,12,13,15,18-20}$ in which the first excited state of the $\mathrm{OH}$-stretching vibration, $|1\rangle$, was prepared by a femtosecond mid-IR pulse and the subsequent VER dynamics were recorded either through the decay of the $|1\rangle$ population as an anharmonically shifted transient absorption to the next higher-lying $\mathrm{OH}$-stretching quantum, $|2\rangle$, or through the recovery of the population of the vibrational ground state, $|0\rangle$, as a transient bleach/stimulated emission on

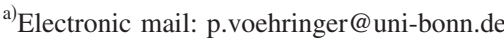

the transition, $|1\rangle \leftrightarrow|0\rangle$. These experiments yielded lifetimes, $\tau_{r}$, which range between 0.5 and 1 ps depending upon the detection frequency. The dependence of $\tau_{r}$ on the probe frequency was interpreted with an intermolecular $\mathrm{O}-\mathrm{H} \cdots \mathrm{O}$ mode acting as the primary energy acceptor for the intramolecular stretching vibration. ${ }^{2,19}$ Such an interpretation is based on the notion that the resonance frequency of an $\mathrm{OH}$ vibrator correlates in a unique fashion with the length of the $\mathrm{H}$ bond to which it is coupled.

Yet, the spectral variance of the excited-state lifetime could in principle also be understood in terms of a distribution of local H-bonded configurations of the excited $\mathrm{OH}$ vibrator, which is inhomogeneous on the VER time scale. Of course, the intermittent breakage and formation of $\mathrm{H}$ bonds interconvert the various $\mathrm{H}$-bonded structures and result in vibrational spectral diffusion throughout the mid-IR $\mathrm{OH}-$ stretching region. From a time-resolved hole-burning experiment, a time scale for spectral diffusion was deduced that was remarkably close to the VER time scale. ${ }^{2}$

To disentangle the two distinctly different processes, VER, on the one hand, and spectral diffusion, on the other, we performed femtosecond mid-IR pump-probe spectroscopy on the OH-stretching mode of HOD in heavy water over wide ranges of pressure and temperature corresponding to the liquid and the supercritical phases of the mixture. A temperature-density diagram, $T(\rho)$, of $\mathrm{D}_{2} \mathrm{O}$ is reproduced in Fig. 1(A), where the stars illustrate the thermodynamic conditions for which the pump-probe experiments reported here were carried out.

The insert in Fig. 1(B) displays linear absorption spectra of the samples for various $(T, \rho)$ tuples corresponding to the open circles inserted in the phase diagram. It can clearly be seen that with increasing temperature and decreasing sample density, the $\mathrm{OH}$-stretching absorption band gradually shifts to higher frequencies. However, it is important to stress that 

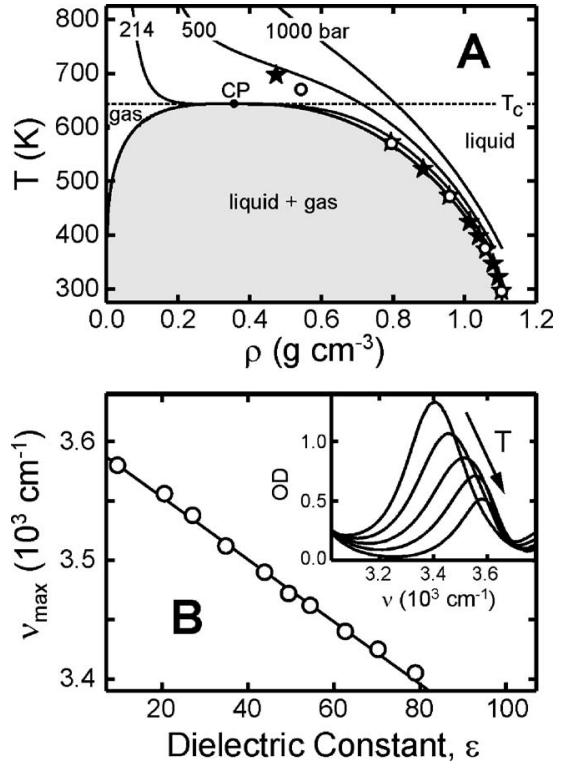

FIG. 1. (A) Temperature-density diagram of $\mathrm{D}_{2} \mathrm{O}$ with selected isobars. $\mathrm{CP}$ marks the critical point. The stars indicate the state points where timeresolved experiments were carried out. (B) Spectral position of the $\mathrm{OH}-$ stretching absorption maximum of HOD vs the solvent dielectric constant. The insert shows corresponding spectra at various pairs of $T$ and $\rho$. Their corresponding thermodynamic state points are indicated in (A) by the open circles. The arrow indicates the order of the spectra with increasing temperature.

$\mathrm{H}$ bonds still exist in the entire $(T, \rho)$ range covered here and, most notably, even under the supercritical conditions which we were able to establish. This is because for the lowest densities, the steady-state spectrum is redshifted by more than $150 \mathrm{~cm}^{-1}$ as compared to its gas-phase counterpart. This conclusion is perfectly in line with previous investigations of the temperature and pressure dependences of the vibrational spectra of water ${ }^{21}$ as well as with recent computer simulations of the structure and the dynamics of liquid-tosupercritical $\mathrm{H}_{2} \mathrm{O}$. ${ }^{22}$

Interestingly, employing a fluctuating charge potential for water and imposing simultaneous geometric and energetic criteria for identifying H-bonded configurations, molecular-dynamics (MD) simulations strongly imply ${ }^{23}$ that the dielectric constant $\varepsilon$ of fluid water is strictly proportional to the average number $n_{\mathrm{HB}}$ of hydrogen bonds per molecule. In other words, the average size of water aggregates that are transiently held together by hydrogen-bonded contacts among the fluctuating monomers can be estimated from the experimental values, $\varepsilon(T, \rho)$, of the density- and temperature-dependent dielectric constants.

Following this idea, the frequency of maximal absorbance, $\nu_{\max }$, of the $\mathrm{OH}$-stretching band is plotted in Fig. 1(B) versus the dielectric constant of water. ${ }^{24}$ Indeed, it exists a remarkable correlation between the two quantities, which can be approximated reasonably well by the simple expression, $\nu_{\max }=3603-2.62(\varepsilon-1) \mathrm{cm}^{-1}$. Provided the $n_{\mathrm{HB}}(\varepsilon)$ correlation found from the MD simulations of Ref. 23 holds true, the absorption spectrum in the $\mathrm{OH}$-stretching region may be viewed as a temporally averaged size distribution of hydrogen-bonded $\mathrm{HOD}-\mathrm{D}_{2} \mathrm{O}$ aggregates. Lowering the sample temperature favors the formation of larger H-bonded
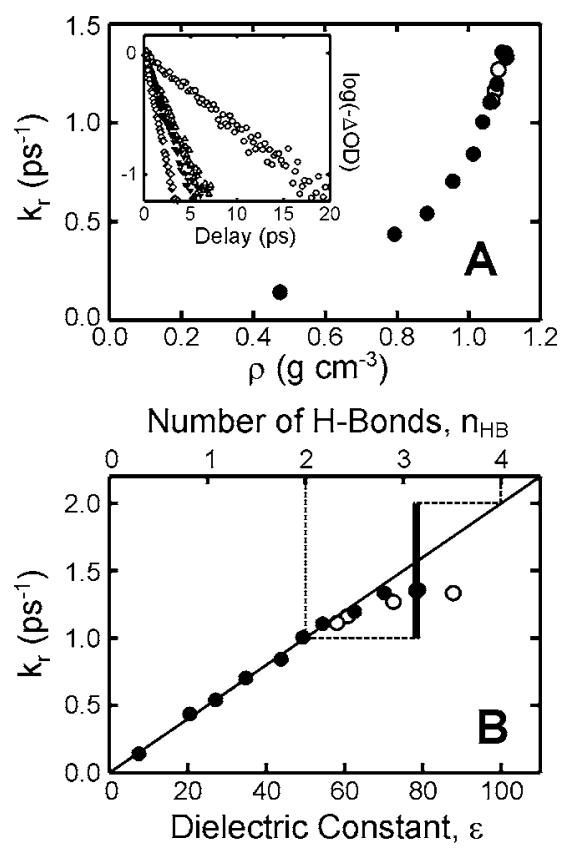

FIG. 2. (A) Density dependence of the $\mathrm{OH}$-stretching relaxation rate constant of HOD in $\mathrm{D}_{2} \mathrm{O}$ (full circles). The open circles are from Refs. 13 and 20. The insert shows normalized transient absorption signals of $2 \%$ HOD in $\mathrm{D}_{2} \mathrm{O}$ for the following thermodynamic conditions: 420 bars and $697 \mathrm{~K}$ (open circles), 100 bars and $572 \mathrm{~K}$ (open triangles), 41 bars and $523 \mathrm{~K}$ (solid triangles), and 7 bars and $424 \mathrm{~K}$ (diamonds). (B) OH-stretching relaxation rate constant of HOD vs the dielectric constant of water. The top axis indicates the average number of hydrogen bonds per water molecule derived from MD simulations (Ref. 23). The thick bar assigns the range of time constants found for ambient conditions (Refs. 5, 12-14, 18, and 20), and the solid line marks the $\varepsilon(T, \rho)$ dependence of the ensemble-averaged VER rate constant

clusters and leads therefore to a gradual redshift of the $\mathrm{OH}$ stretching band. Note that previous studies on the structure and the dynamics of liquid water interpreted the $\mathrm{OH}-$ stretching band as a dynamically averaged length distribution of $\mathrm{D}_{2} \mathrm{O} \cdots \mathrm{HOD}$ bridges. ${ }^{2,19}$ This is because in general, the frequency of $\mathrm{OH}$ vibrators engaged in $\mathrm{O} \cdots \mathrm{H}-\mathrm{O}$ hydrogen bonds decreases with decreasing $\mathrm{O} \cdots \mathrm{O}$ distance. Therefore, heating the liquid (say, under a given pressure) results in a decreasing density, thus to an on-average lengthening of the $\mathrm{H}$ bonds, and hence to a blueshift of the $\mathrm{OH}$-stretching band. At this stage, it is sufficient to emphasize that even without knowing in detail the origin of the perfectly linear $\nu_{\max }(\varepsilon)$ correlation, the dielectric constant, $\varepsilon(T, \rho)$, is a remarkably useful quantity for characterizing the hydrogen-bond status of the OH-stretching vibration of HOD in the liquid-tosupercritical $\mathrm{D}_{2} \mathrm{O}$.

Several pump-probe transients for representative pairs of $T$ and $\rho$ are displayed in Fig. 2(A) (see inset), where the negative pump-induced optical density is plotted semilogarithmically versus the pump-probe time delay. The displayed data were taken with a pump frequency of $3550 \mathrm{~cm}^{-1}$ and a probe frequency of $3540 \mathrm{~cm}^{-1}$ located in the transient bleach region of the $|1\rangle \leftrightarrow|0\rangle$ transition. The excitation bandwidth was $120 \mathrm{~cm}^{-1}$ and the relative pump-probe polarization was set to the magic angle. The sample composition was $2 \%$ $\mathrm{HOD}$ in $\mathrm{D}_{2} \mathrm{O}$. Within our time resolution of $200 \mathrm{fs}$, the ground-state bleach was found to recover strictly monoexpo- 
nentially at temperatures above $400 \mathrm{~K}$. Under these conditions the anharmonically redshifted transient absorption on the $|2\rangle \leftrightarrow|1\rangle$ transition (data not shown) behaves as a mirror image of the transient bleach and yields within the experimental accuracy identical time constants. Furthermore, an isosbestic probe frequency could always be identified which separated the spectral regions of bleach recovery and transient absorption decay and for which no dynamic evolution was detectable. This finding together with the singleexponential behavior and the mirror image correspondence of transient bleach and transient absorption strongly suggests that following the initial mid-IR excitation, the excited $\mathrm{OH}-$ stretching state decays back to the ground vibrational level without appreciably populating any intermediate states such as those arising from the bending vibration. Of course, such an interpretation is incorrect if the absorption cross section for the stretching combination tone of the intermediate state accidentally coincides with the stretching fundamental, $|1\rangle \leftrightarrow|0\rangle$, for all probe frequencies. The observation of isosbestic points above $400 \mathrm{~K}$ further suggests that under these conditions spectral diffusion is much faster than vibrational relaxation and that it proceeds on considerably shorter time scales than at room temperature.

The rate constants, $k_{r}=1 / \tau_{r}$, for vibrational relaxation obtained by fitting single-exponential decays to the data are plotted in Fig. 2(A) as a function of the sample density. Their error is estimated to be about 5\%; above the critical temperature, the error increases to $\sim 10 \%$. For comparison, the temperature-dependent rate constants obtained by Bakker and co-workers ${ }^{13,20}$ for the liquid under ambient pressure are reproduced by the open symbols. The agreement between the two independent data sets at high liquid densities is very good, thereby lending further credence to the more intricate experiments at elevated $p$ and $T$. The overall density dependence is highly nonlinear. The rate constant changes by a factor of 5 (from 0.14 to $0.70 \mathrm{ps}^{-1}$ ) upon doubling the density from $0.475 \mathrm{~g} \mathrm{~cm}^{-3}$ (at $697 \mathrm{~K}$ and 420 bars) to $0.96 \mathrm{~g} \mathrm{~cm}^{-3}$ (at $473 \mathrm{~K}$ and 19 bars). Note, however, that there are two effects superimposed.

The first effect is related to the temperature dependence of the frequency of solute-solvent encounters. Adapting the (certainly oversimplified) isolated binary collision scenario from early studies of VER in nonpolar nonassociating liquids, this frequency should scale linearly with $T^{1 / 2}$ (at constant density). However, this pure dynamical affect is apparently more than compensated by the rapid buildup of a complete first solvation shell around the vibrationally excited HOD on cooling the fluid to below the supercritical temperature.

Following the above lines of arguments, the VER rate constant is plotted in Fig. 2(B) as a function of the temperature- and density-dependent dielectric constants. Using the results from the MD simulations of Ref. 23, the latter quantity is translated into an average number of $\mathrm{H}$ bonds, $n_{\mathrm{HB}}$, as depicted by the top horizontal axis. For comparison, the data taken in the region of thermodynamic stability of the liquid under ambient pressure ${ }^{13}$ are shown as the open circles. Once again, there is an astonishing linear relationship between $k_{r}$ and $\varepsilon(T, \rho)$, both of which are independently determined experimental quantities. Only for $\varepsilon(T, \rho)>70$, the data seem to level off at a constant value for $k_{r}$ of $1.4 \mathrm{ps}^{-1}$. As evidenced by the straight line, the data in the interval $1 \leqslant \varepsilon(T, \rho) \leqslant 70$ can be very well represented by the simple relation $k_{r}=B(\varepsilon-1)$ where the slope $B$ equals $(50 \mathrm{ps})^{-1}$.

As mentioned above, the excited-state lifetime, $\tau_{r}$, at room temperature and 1 bar varied between 0.5 and 1 ps depending upon the detection scheme, the probe wavelength, and the analysis method. ${ }^{5,12-14,18,20}$ This variation corresponds to a range of vibrational relaxation rates under ambient conditions, which is symbolized in Fig. 2(B) by the vertical bar. It was suggested in a recent mid-IR-pump/antiStokes-probe study by Wang et $a .^{5}$ that VER is slower for a subensemble of weakly hydrogen-bonded $\mathrm{OH}$ oscillators absorbing on the blue edge of the steady-state absorption band $\left(\tau_{r} \sim 0.8 \mathrm{ps}\right)$ as compared to a subensemble engaged in stronger hydrogen bonds that were excited on the red edge $\left(\tau_{r}\right.$ $\sim 0.5 \mathrm{ps}$ ). According to Fig. 1(B), the latter structures can be characterized by higher values of $\varepsilon(T, \rho)$ as compared to the blue-absorbing species. Hence, using the alternative "cluster" notion and the $n_{\mathrm{HB}}(\varepsilon)$ correlation from the MD simulations, the slowly relaxing species correspond to local solute-solvent structures that involve an average of two $\mathrm{H}$ bonds only. Likewise, the faster components can be attributed to local structures connected by four hydrogen bonds (including, e.g., the famous Walrafen pentamer). Such phenomenological assignments are illustrated in Fig. 2(B) by the dashed lines. Yet, one has to bear in mind that different H-bonded structures can interconvert on the time scale of hydrogen-bond breakage and formation, a process which gives rise to spectral diffusion and which is believed to occur on a time scale similar to that of VER under ambient conditions. ${ }^{2-4,13-15,17-19,25}$

Assuming for the moment that spectral diffusion occurs much faster than VER, the measured rate is independent of the frequency of the pump and probe pulses. It simply corresponds to a weighted average over all possible H-bonded structures, each of which can relax with its proper (clusterspecific) VER rate. For a given pair of $T$ and $\rho$, the statistical weight of each component is given by its appropriate thermodynamic abundance. In fact, this is also how the top horizontal axis should be interpreted: the precise value of $n_{\mathrm{HB}}$ corresponds to the ensemble-averaged number of $\mathrm{H}$ bonds formed between the solute HOD and the solvent $\mathrm{D}_{2} \mathrm{O}$. With the exception of our low-temperature, low-pressure data, a probe-frequency dependence was never unambiguously observed within the experimental accuracy. Consequently, the two ensemble-averaged quantities, $k_{\mathrm{VER}}$ and $n_{\mathrm{HB}}$, correlate linearly as expected.

In the other limit, however, where spectral diffusion is much slower than VER, $n_{\mathrm{HB}}$ is still equal to the ensembleaveraged number of $\mathrm{H}$ bonds, however, one can now expect the measured rate to critically depend on the frequencies of the pump and probe pulses. This is, e.g., because the frequency of the pump pulse selects a specific subensemble out of all possible $\mathrm{H}$-bonded structures, which is not allowed to convert into all the others on the time scale of vibrational 
relaxation. Hence, the observed rate corresponds to a clusterspecific rate, i.e., a value which is highly characteristic for a selected H-bonded configuration. Again, we reemphasize that the terms "cluster size" and "hydrogen-bond length" are equally (in)appropriate as both phenomena are experimentally indistinguishable at this stage.

According to the above discussion, we interpret the deviation of the measured relaxation rate constants from the straight line, $k_{r}=B(\varepsilon-1)$, by nonseparable time scales of spectral diffusion and vibrational relaxation. For $\varepsilon$ values beyond 70, the experimental values for $k_{r}$ reported in the previous literature begin to scatter quite naturally simply because the $\mathrm{OH}$-stretching absorption band becomes increasingly inhomogeneous on the time scale of VER. An ensemble-averaged value for the rate constant at room temperature and 1 bar can, however, be specified by extrapolating on the straight line of Fig. 2(B) to $\varepsilon\left(298 \mathrm{~K}, 1.1 \mathrm{~g} \mathrm{~cm}^{-1}\right)$, which gives $k_{r}=1.58 \mathrm{ps}^{-1}$. Provided VER proceeds either directly back to the ground vibrational level or sequentially to $|0\rangle$ without creating substantial transient populations in intermediate states, this value is consistent with a lifetime of the first excited $\mathrm{OH}$-stretching state of $\mathrm{HOD}$ in $\mathrm{D}_{2} \mathrm{O}$ of $0.63 \mathrm{ps}$.

In summary, we have presented femtosecond mid-IR pump-probe experiments on $\mathrm{OH}$-stretching vibrational relaxation of $\mathrm{HOD}$ in $\mathrm{D}_{2} \mathrm{O}$ over a wide range of temperature and density. Using a phenomenological correlation between the observed rate constant and the dielectric constant of the solvent, we were able to differentiate between the dynamics of energy relaxation and spectral diffusion brought about by hydrogen-bond breakage and formation. The inconsistencies between different experimental techniques previously employed to study VER in the liquid under ambient conditions are due to an accidental overlap of time scales of these two distinct dynamical processes in the hydrogen-bonded network. We hope that the results reported here will serve as a suitable benchmark for future theoretical studies of vibrational energy relaxation in this most fascinating fluid.
This work was supported by the Deutsche Forschungsgemeinschaft through the SFB 357 "Molekulare Mechanismen unimolekularer Prozesse" and Grant No. VO 593/4-1.

${ }^{1}$ Water: A Comprehensive Treatise, edited by F. Franks (Plenum, New York, 1972).

${ }^{2}$ H. J. Bakker, H.-K. Nienhuys, G. Gallot, N. Lascoux, N. G. M. Gale, J.-C. Leicknam, and S. Bratos, J. Chem. Phys. 116, 2592 (2002).

${ }^{3}$ S. Woutersen, U. Emmerichs, and H. J. Bakker, Science 278, 658 (1997).

${ }^{4}$ E. T. J. Nibbering and T. Elsaesser, Chem. Rev. (Washington, D.C.) 104, 1887 (2004)

${ }^{5}$ Z. Wang, A. Pakoulev, Y. Pang, and D. D. Dlott, J. Phys. Chem. A 108, 9054 (2004)

${ }^{6}$ K. Winkler, J. Lindner, and P. Vöhringer, Phys. Chem. Chem. Phys. 4, 2144 (2002)

${ }^{7}$ E. W. Castner, Y. J. Chang, Y. C. Chu, and G. E. Walrafen, J. Chem. Phys. 102, 653 (1995).

${ }^{8}$ S. Palese, S. Mukamel, R. J. D. Miller, and W. T. Lotshaw, J. Phys. Chem. 100, 10380 (1996).

${ }^{9}$ R. Torre, P. Bartolini, and R. Righini, Nature (London) 428, 296 (2004).

${ }^{10}$ M. L. Cowan, B. D. Bruner, N. Huse, J. R. Dwyer, B. Chugh, E. T. J. Nibbering, T. Elsaesser, and R. J. D. Miller, Nature (London) 434, 199 (2005).

${ }^{11}$ C. J. Fecko, J. J. Loparo, S. T. Roberts, and A. Tokmakoff, J. Chem. Phys. 122, 054506 (2005).

${ }^{12}$ R. Laenen, C. Rauscher, and A. Laubereau, Phys. Rev. Lett. 80, 2622 (1998).

${ }^{13}$ S. Woutersen, U. Emmerichs, H.-K. Nienhuys, and H. J. Bakker, Phys. Rev. Lett. 81, 1106 (1998).

${ }^{14}$ G. M. Gale, G. Gallot, and N. Lascoux, Chem. Phys. Lett. 311, 123 (1999).

${ }^{15}$ A. J. Lock and H. J. Bakker, J. Chem. Phys. 117, 1708 (2002).

${ }^{16}$ O. F. A. Larsen and S. Woutersen, J. Chem. Phys. 121, 12143 (2004).

${ }^{17}$ N. Huse, S. Ashihara, E. T. J. Nibbering, and T. Elsaesser, Chem. Phys. Lett. 404, 389 (2005).

${ }^{18}$ G. M. Gale, G. Gallot, F. Hache, N. Lascoux, S. Bratos, and J.-C. Leicknam, Phys. Rev. Lett. 82, 1068 (1999).

${ }^{19}$ S. Bratos, G. M. Gale, G. Gallot, F. Hache, N. Lascoux, and J.-C. Leicknam, Phys. Rev. E 61, 5211 (2000).

${ }^{20}$ H.-K. Nienhuys, S. Woutersen, R. A. van Santen, and H. J. Bakker, J. Chem. Phys. 111, 1494 (1999).

${ }^{21}$ Y. E. Gorbaty and A. G. Kalinichev, J. Phys. Chem. 99, 5336 (1995).

${ }^{22}$ M. Boero, K. Terakura, T. Ikeshoji, C. C. Liew, and M. Parrinello, J. Chem. Phys. 115, 2219 (2001).

${ }^{23}$ N. Yoshii, S. Miura, and S. Okazaki, Chem. Phys. Lett. 345, 195 (2001).

${ }^{24}$ The dielectric constant as a function of $p$ and $T$ was obtained from PROPATH Group, PROPATH, a program package for thermophysical properties, version 12.1 (2001).

${ }^{25}$ S. Yeremenko, M. S. Pshenichnikov, and D. A. Wiersma, Chem. Phys. Lett. 369, 107 (2003). 